\title{
The Rap-B-Raf signalling pathway is activated by glucose and glucagon-like peptide-1 in human islet cells
}

Received: 16 December 2004 / Accepted: 20 March 2005 / Published online: 2 July 2005

(C) Springer-Verlag 2005

\begin{abstract}
Aims/hypothesis: Glucose and glucagon-like peptide-1 have been shown to activate extracellular signalregulated kinase (ERK) and phosphoinositide 3-kinase in beta cells. We examined the contributions of the small GTPases Rap and Ras and the serine-threonine kinases BRaf and Raf- 1 to the activation of these kinases in human islet cells. Methods: The expression of Rap, Ras, B-Raf and Raf-1 in human islets was examined by immunohistochemistry and immunoblotting. Human islets were incubated in glucose at concentrations of 2.5 and $15 \mathrm{mmol} / \mathrm{l}$ and were stimulated with $10 \mathrm{nmol} / 1$ glucagon-like peptide- 1 . The activation of ERK and Raf kinases was examined by phosphorylation-specific antibodies and immuno-complexed kinase assays. The activation of Rap and Ras was determined by pull-down assays. Stimulation of phosphoinositide 3-kinase was detected by immuno-complexed lipid kinase assays. Results: Extracellular-regulated kinase and protein kinase B (a downstream target of phosphoinositide 3-kinase) were activated in islets stimulated with glucose and glucagon-like peptide-1. In these islets, the Rap-B-Raf
\end{abstract}

J. Trümper and D. Ross contributed equally to this article

J. Trümper · D. Ross · R. Göke · D. Hörsch

Department of Internal Medicine,

Division of Gastroenterology and Endocrinology,

Philipps University of Marburg,

Marburg, Germany

H. Jahr · M. D. Brendel

Department of Internal Medicine,

Division of Endocrinology,

Justus Liebig University of Giessen,

Giessen, Germany

D. Hörsch $(\bowtie)$

Department of Internal Medicine,

Division of Gastroenterology and Metabolism,

Philipps University of Marburg,

Baldingerstrasse,

35033 Marburg, Germany

e-mail: hoerschd@med.uni-marburg.de

Tel.: +49-6421-2862780

Fax: +49-6421-2868922 signalling pathway was activated preferentially compared with Ras and Raf-1, and activated Rap and B-Raf mediated ERK stimulation in kinase assays in vitro. In addition, Rap rather than Ras mediated activation of phosphoinositide 3kinase in islets stimulated with glucose and glucagon-like peptide-1. Conclusions/interpretation: In human islet cells, glucose and glucagon-like peptide-1 activate the Rap and BRaf signalling module, which mediates ERK activation in assays in vitro. Rap also activates phosphoinositide 3-kinase, delineating central roles for Rap and B-Raf as therapeutic targets for beta cell growth in diabetes mellitus.

Keywords Beta cell - Extracellular signal-regulated kinase - Glucagon-like peptide-1 · Phosphoinositide 3-kinase $\cdot$ Small GTPase

Abbreviations DAB: diaminobenzidine - ERK: extracellular signal-regulated kinase - GDS: GDP dissociation stimulator - GEF: guanine nucleotide exchange factors - GLP-1: glucagon-like peptide-1 - GST: glutathione $S$-transferase - MAPK: mitogen-activated protein kinase MBP: myelin basic protein - MEK: mitogen-activated protein kinase kinase $\cdot \mathrm{PI} 3 \mathrm{~K}$ : phosphoinositide 3-kinase · PKB: protein kinase $\mathrm{B} \cdot \mathrm{RBD}$ : Ras-binding domain

\section{Introduction}

The glucoincretin hormone glucagon-like peptide-1 (GLP-1) potentiates glucose-induced insulin secretion and activates mitogenic kinases such as phosphoinositide 3-kinase (PI3K) and mitogen-activated protein kinase (MAPK), also known as extracellular signal-regulated kinase (ERK) [1]. ERK is typically stimulated by the sequential activation of small GTPases (such as Ras), Raf kinases and mitogen-activated protein kinase kinase (MEK). In endocrine cells, the small GTPase Rap and the serine-threonine kinase B-Raf kinase regulate ERK signalling as opposed to the prototypical Ras and Raf-1 kinase pathway [2].

Glucose and GLP-1 have been shown to activate ERK in numerous beta cell lines and in murine islets [3-5]. How- 
ever, the published results are inconsistent with respect to whether this activation is dependent on [3] or independent of $[4,5]$ Ras and Raf kinases. These discrepancies may be attributed to the different beta cell lines used in the different studies. The aim of the present study was to examine the expression and activation of GTPases and Raf kinases by glucose and GLP-1 in isolated human islets [6], based on the fact that human beta cells are essential targets for beta cell growth strategies in diabetes mellitus.

\section{Materials and methods}

Materials GLP-1 (7-36) and IGF-1 were from Bachem (Bubendorf, Switzerland). New England Biolabs (Beverly, MA, USA) supplied antibodies directed against protein kinase B (PKB) and phosphorylated PKB. Antibodies for phosphorylated ERK-1/2 (pERK $\mathrm{Tyr}^{204}$ ), ERK-2, Ras, Rap1, Raf-1 and B-Raf were from Becton Dickinson (Heidelberg, Germany) and Santa Cruz Biotechnology (Santa Cruz, CA, USA). The proteins Raf-1 Ras-binding domain (Raf-1-RBD) and Ral GDP dissociation stimulator Ras-binding domain (Ral-GDS-RBD) were purified as glutathione $S$-transferase (GST) fusion proteins. Myelin basic protein (MBP), inactive GST-MEK-1, inactive GSTERK-2, active Raf, and phospho-MBP antibodies and control MBP antibodies were from Upstate Biotechnology (Waltham, MA, USA).

Cell culture Islets were isolated from human pancreases obtained from six brain-dead multi-organ donors after obtaining legal consent. Approval from the local ethics committee was obtained. The islets were isolated at the Giessen Islet Isolation and Transplantation Centre (Giessen, Germany) according to a modified semi-automated digestion-filtration method and cultured as described previously $[6,7]$. Human islet cells were stimulated with glucose for $4 \mathrm{~h}$, since shorter incubation times yielded unreliable results for ERK and PI3K activation, while longer incubations increased the expression of signalling intermediates such as ERK and PKB.

Immunohistochemistry Sections of normal human pancreas were examined for expression of Ras, Rap, B-Raf and Raf-1 during profiling of endocrine pancreatic tumours (approval from the local ethics committee was obtained). Immunohistochemistry was performed using monoclonal and polyclonal antibodies by means of the standard avidin-biotin-peroxidase complex (ABC) method ( $\mathrm{ABC}$ Elite Kit; Vector, Burlingame, CA, USA) using 3,3'diaminobenzidine (DAB) as the chromogen after microwave pretreatment performed by heating the deparaffinised and rehydrated sections, immersed in $10 \mathrm{mmol} / 1$ sodium citrate buffer ( $\mathrm{pH} 6.0$ ), in a microwave oven at $600 \mathrm{~W}$ for $5 \mathrm{~min}$, three times. Sections were counterstained using Mayer's hemalum. After dehydration in graded alcohols, sections were cleared in xylene and coverslipped with Entellan (Merck, Darmstadt, Germany).
Immunocomplexed Raf kinase assays Raf kinase assays were performed with immunoprecipitated proteins incubated with inactive GST-MEK-1 and GST-ERK-2 for $30 \mathrm{~min}$ at $30^{\circ} \mathrm{C}$. The reaction was terminated by the addition of EDTA. The activity of GST-ERK-2 was determined using either MBP as a substrate or by immunoblotting an aliquot with antibodies to phospho-ERK-1/2.

Pull-down assays for the isolation of active Ras and Rap Cellular lysates were subjected to pull-down with sepharose beads bound to a GST fusion protein of Raf-1-RBD for the detection of active Ras, and beads bound to a GST fusion protein of Ral-GDS-RBD for active Rap [8]. Raf kinases were immunoprecipitated. After extensive washing of the samples in lysis buffer, proteins were separated by SDS-PAGE, and GTP-bound active Ras and Rap were detected by immunoblotting.

Phosphoinositide 3-kinase assays Immunocomplexed and cellular PI3K activity was determined as previously described [9]. In brief, immunocomplexes or cellular lysates were incubated in a 55- $\mu$ l reaction mixture containing $440 \mu \mathrm{mol} / 1 \mathrm{ATP}, 185 \mathrm{kBq}\left[\gamma_{-}{ }^{32} \mathrm{P}\right] \mathrm{ATP}, 10 \mathrm{mmol} / 1$ $\mathrm{MgCl}_{2}$ and $5 \mu \mathrm{g}$ phosphatidylinositol for $20 \mathrm{~min}$ at room temperature. Reactions were stopped by the addition of $20 \mu \mathrm{l}$ $8 \mathrm{~N} \mathrm{HCl}$ and $160 \mu \mathrm{l}$ chloroform/methanol $(1: 1 \mathrm{v} / \mathrm{v})$. The organic phase was removed by centrifugation and applied to silica gel thin-layer chromatography plates, which were developed in $\mathrm{CHCl}_{3}: \mathrm{CH}_{3} \mathrm{OH}: \mathrm{H}_{2} \mathrm{O}: \mathrm{NH}_{4} \mathrm{OH}(60: 47: 11.3: 2)$, dried, and then visualised by autoradiography.

\section{Results}

The expression of the GTPases Ras and Rap1 and the serine-threonine kinases Raf-1 and B-Raf in human islets was examined by immunoblotting cellular lysates of isolated human islets (Fig. 1a-d) and by immunohistochemistry (Fig. 1e,f) on paraffin sections of normal human pancreas. B-Raf (Fig. 1c) and Raf-1 (Fig. 1d) were found to be present in multiple splice forms whereas Rap1 (Fig. 1a) and Ras (Fig. 1b) were expressed as single bands at $21 \times 10^{3} \mathrm{M}_{\mathrm{r}}$. Immunohistochemistry of normal pancreas revealed that Rap1 (Fig. 1e) and B-Raf (Fig. 1f) were expressed in human islets. Exocrine acinar cells were also immunopositive for Rap1 (Fig. 1e); in contrast, B-Raf (Fig. 1f) was confined to the endocrine compartment of the pancreas. Few of the cells in the human islets were strongly Rap-positive (Fig. 1e). Compared with Rap expression, BRaf expression was more homogeneous (Fig. 1f). The strongly Rap-positive cells represent insulin-expressing beta cells and somatostatin-expressing delta cells (D. Karhoff [Philipps University Marburg, Germany] and D. Hörsch, unpublished results). The expression of Ras and Raf-1 was ubiquitous - it was detected in every cell type of exocrine and endocrine pancreas (data not shown).

ERK-1/2 in human islets was activated in the presence of $15 \mathrm{mmol} / 1$ glucose plus $10 \mathrm{nmol} / \mathrm{l} \mathrm{GLP}-1$, as revealed by phosphorylation at tyrosine 204, albeit at lower levels than 
Fig. 1 Expression of Rap1

(a), Ras (b), B-Raf (c) and Raf-1 (d) in cellular lysates of isolated human islets by immunoblotting. Isolated human islets were lysed, separated by SDS-PAGE and immunblotted with the indicated antibodies. The arrows denote non-specific bands. BRaf was present in $95 \times 10^{3}$ and $70 \times 10^{3} \mathrm{M}_{\mathrm{r}}$ splice forms (c) and Raf- 1 in $75 \times 10^{3}$ and $66 \times 10^{3} \mathrm{M}_{\mathrm{r}}$ splice forms (d). Microanatomy of Rap1 (e) and B-Raf (f) in human islets of normal pancreas revealed by immunohistochemistry on paraffin sections. Deparaffinised sections were probed with antibodies to Rap1 and B-Raf using diaminobenzidine as the chromogen

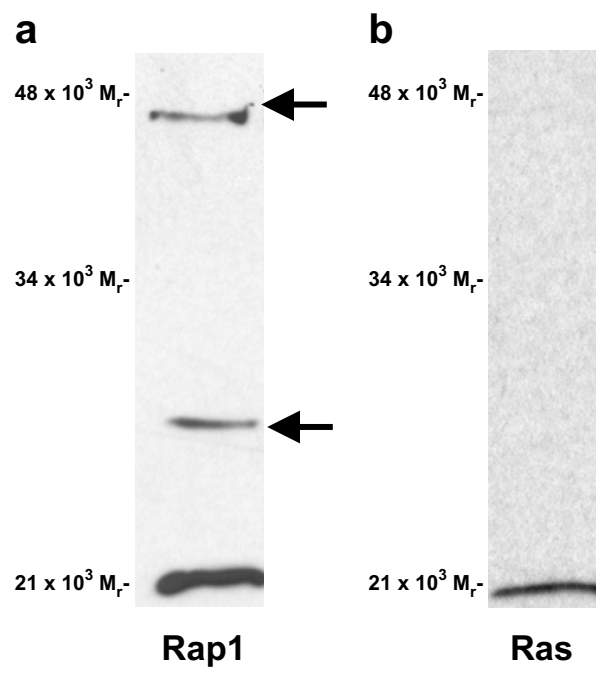

Rap1

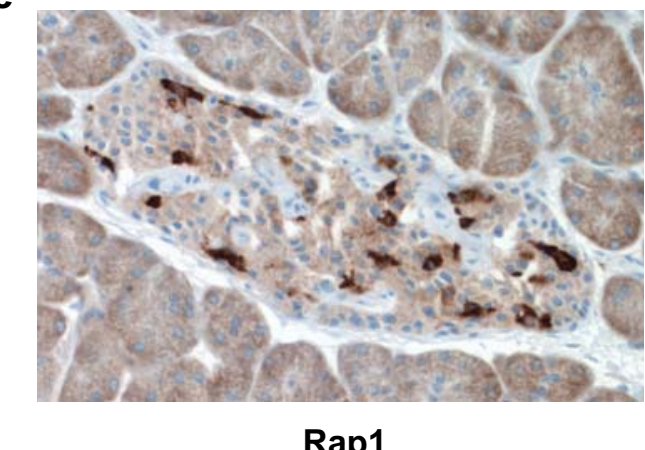

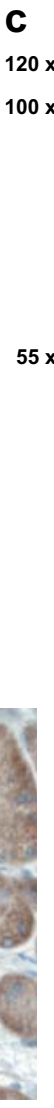

C

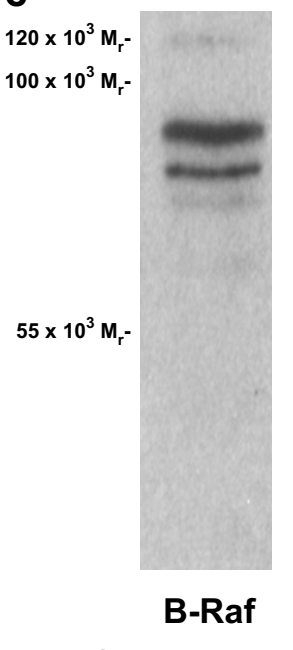

f

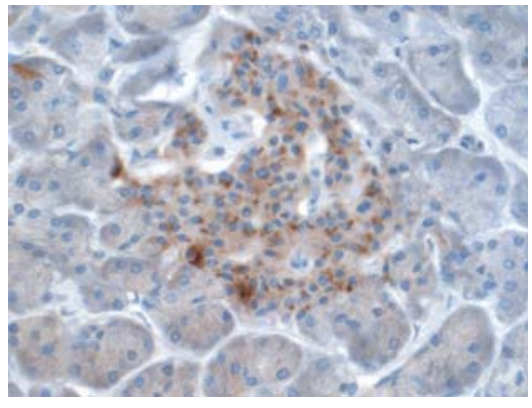

B-Raf d

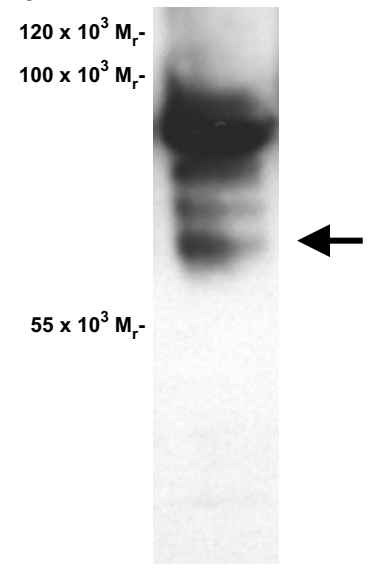

Raf-1 after the addition of $15 \mathrm{mmol} / \mathrm{l}$ glucose plus $10 \mathrm{nmol} / \mathrm{l} \mathrm{IGF-}$ 1 (Fig. 2a). We next examined the ability of Rap1, Ras, BRaf and Raf-1 to stimulate ERK activity in vitro using immunocomplexed Raf kinase assays with inactive GSTMEK and GST-ERK-2. Phosphorylated ERK-2 and MPB were used as references (Fig. 2b,c). Raf kinase assays with and without active Raf were used as positive and negative controls, respectively (Fig. 2b). The results revealed that Raf kinase activity leading to ERK activation in islets stimulated with $15 \mathrm{mmol} / 1$ glucose and $10 \mathrm{nmol} / \mathrm{l} \mathrm{GLP}-1$ was associated with immunoprecipitated Rap and B-Raf (Fig. 2b) rather than Ras and Raf-1 (Fig. 2c). Ras immunoprecipitations revealed only a low increase in the phosphorylation of ERK-2 and MBP, and Raf-1 immunoprecipitates showed no increase in ERK-2 phosphorylation and a low increase in MBP phosphorylation (Fig. 2c). These observations indicate the minor activation of Ras and no stimulation of Raf-1 kinase by glucose and GLP-1 in islets.

The activation of ERK by Raf kinases is preceded by the binding of small G-proteins such as Rap to B-Raf, or Ras to Raf-1. The binding of active Rap to B-Raf was demonstrated by pull-down assays of cellular lysates of islets stimulated with $15 \mathrm{mmol} / \mathrm{l}$ glucose and/or $10 \mathrm{nmol} / \mathrm{l} \mathrm{GLP}-1$ with a GST fusion protein encompassing the RBD of RalGDS [8] or immunoprecipitated B-Raf. The binding of active Ras to Raf-1 was shown by pull-down assays with a GST fusion protein encompassing the RBD of Raf-1. Pull-downs of Rap and Ras performed in the presence of GTP $\gamma \mathrm{S}$ and GDP served as positive and negative controls, respectively (Fig. 3a,b). The binding of (active) Rap to the GST fusion protein of Ral-GDS-RBD and to BRaf was stimulated by glucose and by GLP-1 (Fig. 3a). In contrast, the binding of active Ras to the GST fusion protein of Raf-1-RBD was only slightly increased by high glucose and by GLP-1 (Fig. 3b).

It has previously been shown that glucose and GLP-1 stimulate PI3K and PKB in insulinoma cell lines such as INS-1 $[10,11]$. Consistent with these results, in the present study we have demonstrated that glucose and GLP-1 induce the phosphorylation of PKB at serine 473 and threonine 308 in lysates of human isolated islets (Fig. 4a). The lipid kinase PI3K is activated by the binding of Ras to RBDs present in catalytic and regulatory subunits [12]. Based on this, we examined whether Ras and Rap activate $\mathrm{PI} 3 \mathrm{~K}$ in human islets stimulated with $15 \mathrm{mmol} / \mathrm{l}$ glucose and/or $10 \mathrm{nmol} / 1$ GLP-1. We performed Ras and Rap immunoprecipitations and determined lipid kinase activity using phosphatidylinositol as a substrate. Glucose- and GLP-1-activated PI3K was associated with Rap but not with Ras (Fig. 4b). Thus, the activation of Rap by glucose and GLP-1 in human islets leads to the stimulation of PI3K in addition to the stimulation of ERK via B-Raf. 
a

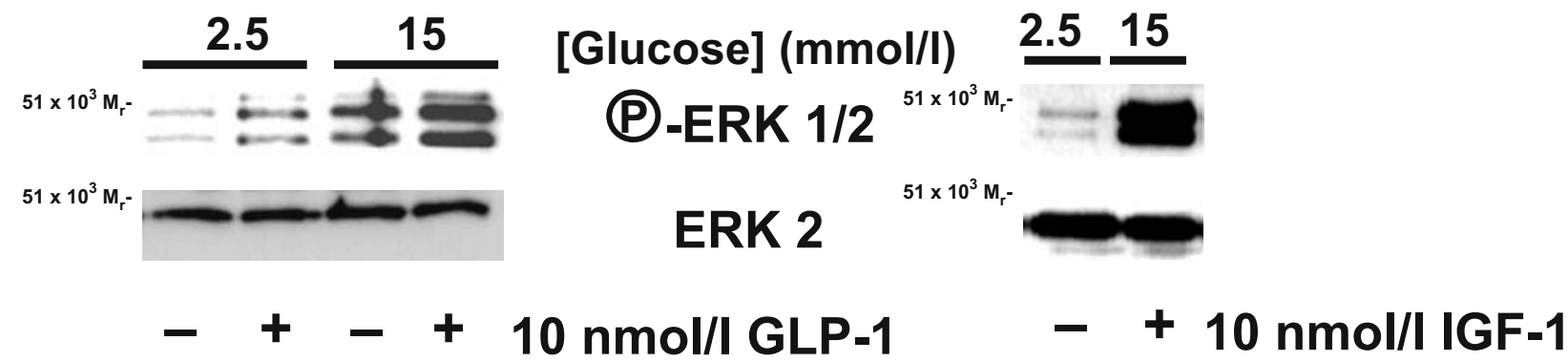

b

\section{$2.5 \quad 15$}

IP: Rap1

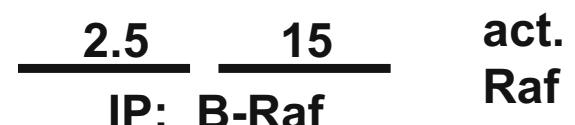

IP: B-Raf

Raf
P-GST-ERK 2

P.-MBP

GST-ERK-2

$71 \times 10^{3} \mathrm{Mr}_{\mathrm{r}}$

$53 \times 10^{3} \mathrm{Mr}-$
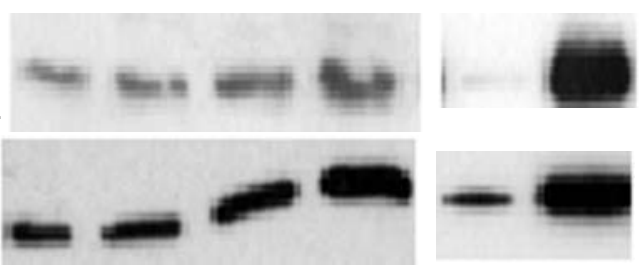

$34 \times 10^{3} \mathrm{Mr}_{\mathrm{r}}$

$21 \times 10^{3} \mathrm{M}_{\mathrm{r}}$

$71 \times 10^{3} \mathrm{M}_{\mathrm{r}^{-}}$

$21 \times 10^{3} \mathrm{Mr}^{-}$

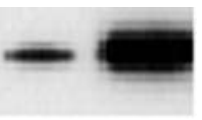

MBP
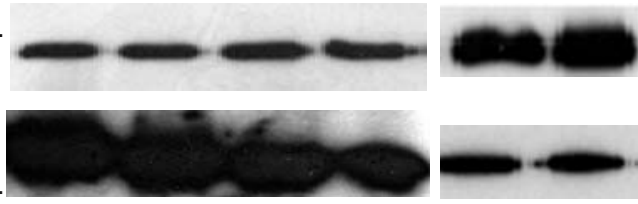

$10 \mathrm{nmol} / \mathrm{l}$ GLP-1

$21 \times 10^{3} \mathrm{Mr}_{\mathrm{r}}$

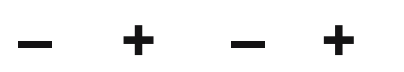

[Glucose] (mmol/l)

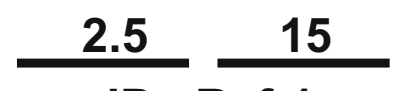

IP: Raf-1

IP: Ras

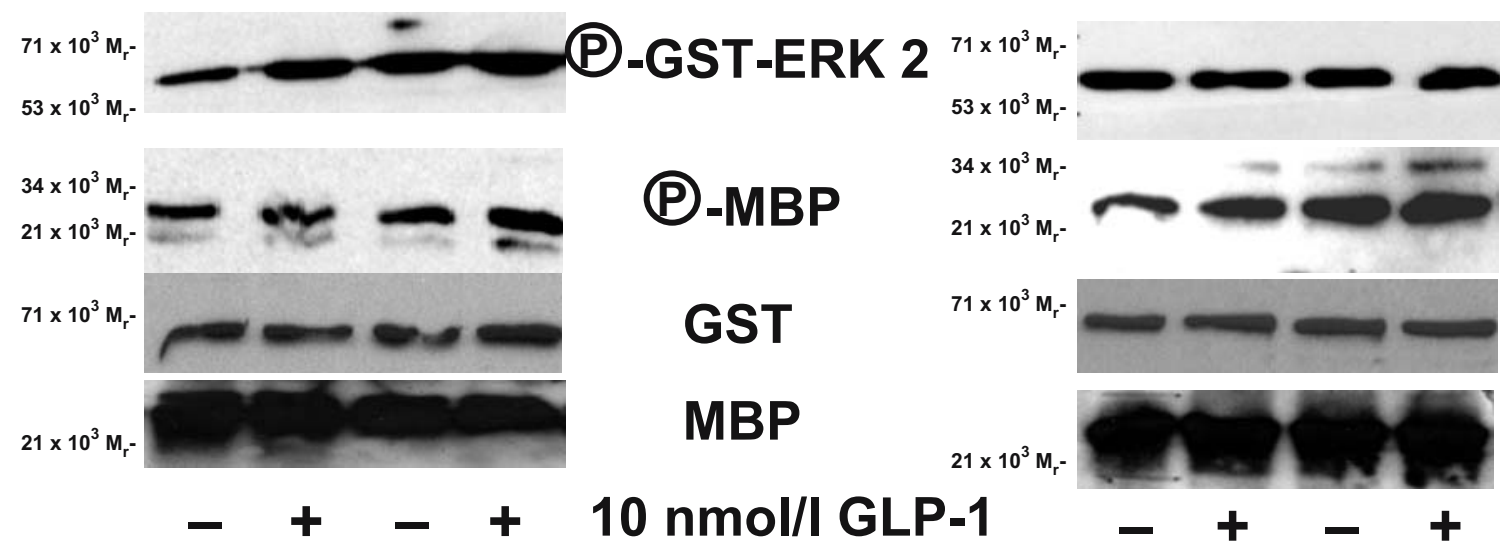

Fig. 2 Glucose and GLP-1 activate ERK and the Rap-B-Raf signalling pathway in isolated human islets. Human islets were equilibrated in 2.5 or $15 \mathrm{mmol} / \mathrm{l}$ glucose for $4 \mathrm{~h}$ and then stimulated with $10 \mathrm{nmol} / 1$ GLP-1 for $10 \mathrm{~min}$. Phosphorylation of ERK (a). This was determined using phosphorylation-specific antibody, and equal loading was confirmed by immunoblotting for non-phosphorylated ERK-2. The blots shown are representative of four different islet preparations. Human islets stimulated with $10 \mathrm{nmol} / 1 \mathrm{IGF}-1$ were used as a control. Immunocomplexed Raf-kinase activation in human islets. Raf-kinase assays were performed with immunoprecipitated Rap and B-Raf (b) or Ras and Raf-1 (c) using inactive GST-MEK-1, GST-ERK-2 and MBP as substrates. Phosphorylation of GST-ERK2 and MBP was determined by immunoblotting with phosphorylation-specific antibodies. Blots of GST-ERK-2 and MBP are included as controls. Shown are representative blots of four different islet preparations. Raf kinase assays using active Raf were used as a positive control and are shown on the right side of panel (b). IP immunoprecipitation 

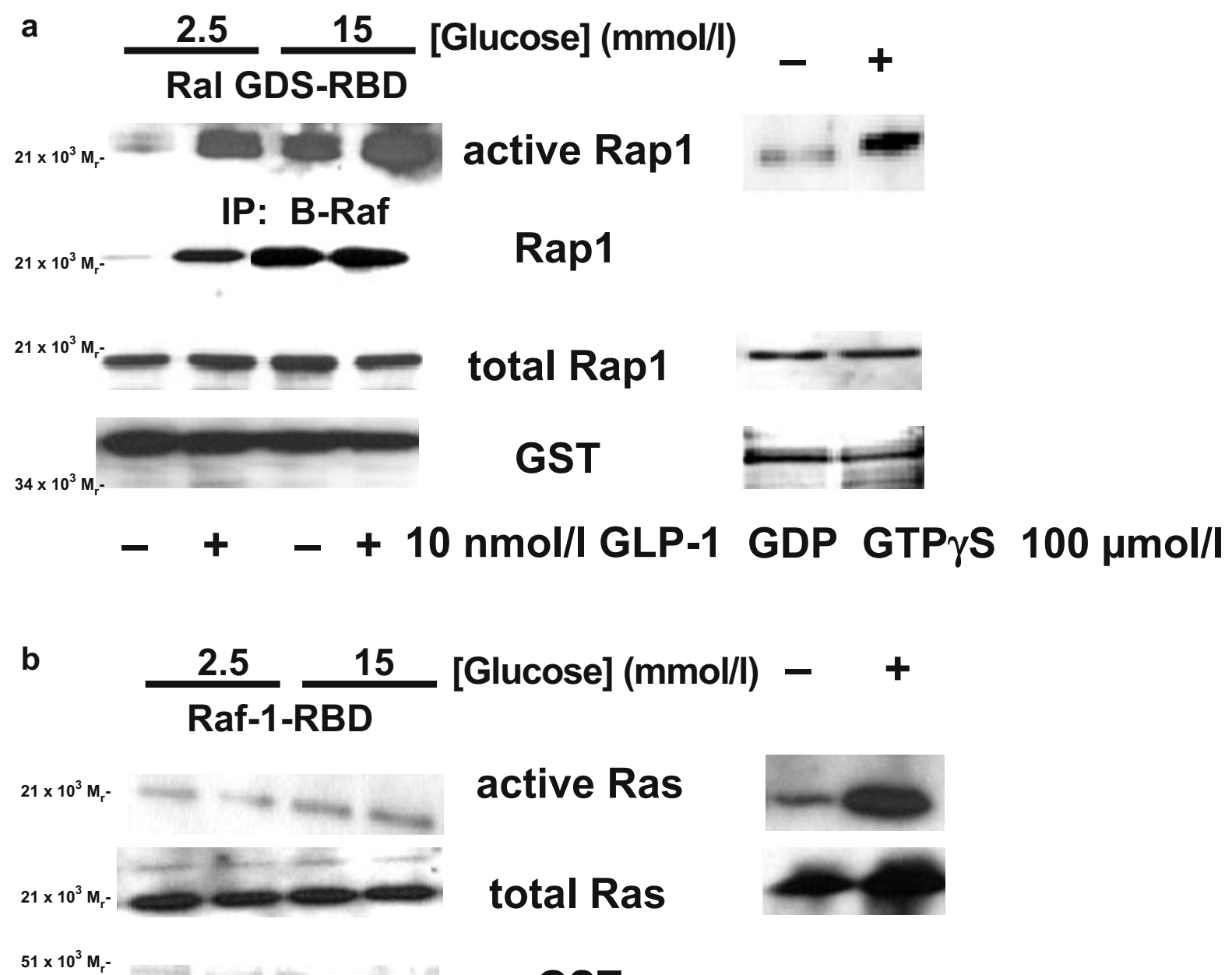

active Ras

total Ras

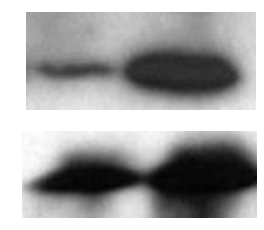

GST

\section{$-\quad+\quad$ + $10 \mathrm{nmol} / \mathrm{l}$ GLP-1 GDP GTP $\gamma \mathrm{S} 100 \mu \mathrm{mol} / \mathrm{l}$}

Fig. 3 In isolated human islets, Rap is activated rather than Ras by glucose and GLP-1 stimulation. Active, GTP-bound Rap was identified by pull-downs of cellular lysates using a GST fusion protein of Ral-GDS-RBD or immunoprecipitated B-Raf (a). Active GTPbound Ras was determined by pull-down with a GST fusion protein of Raf-1-RBD (b). Active Rap and Ras were subsequently detected by immunblotting. The lower panels of each series of blots depict

\section{Discussion}

MAPK activation is pivotal for cellular functions, such as differentiation and growth, in insulin-producing beta cells. In the present study we used purified human islets to examine signalling by Rap and B-Raf in beta cells, based on the fact that these cells are targets for future cell growth strategies aimed at developing treatments for diabetes [1]. Although these cell preparations are not pure, beta cells are selectively responsive to stimulation with glucose and GLP-1, indicating that our results may actually underestimate the degree of ERK and PI3K activation by glucose and GLP-1.

We have demonstrated that, in isolated islets stimulated with $15 \mathrm{mmol} / 1$ glucose plus $10 \mathrm{nmol} / \mathrm{l} \mathrm{GLP}-1$, the GTPase Rap and the serine-threonine kinase B-Raf are activated as internal controls for the GST fusion protein Ral-GDS-RBD with anti-GST antibodies and the expression of Rap (a) or Ras (b) using equal amounts of cellular lysates. In addition, the panels on the right-hand side show positive and negative controls using $100 \mu \mathrm{mol} / 1$ GTP $\gamma \mathrm{S}$ and GDP, respectively. The blots shown are representative of four different islet preparations. IP immunoprecipitation

opposed to Ras and Raf-1, which are also expressed in human beta cells. During the course of our study, we noted that in differentiated rodent beta cell lines, specifically, certain INS-1 cell lines and RinM5F cells (D. Hörsch, unpublished results), Rap and B-Raf are not always activated by glucose and GLP-1. This may be due to the altered glucose responsiveness of these cells, and may explain some of the conflicting results obtained with respect to the activation of GTPases and Raf kinases in beta cells [3-5].

Although, we demonstrated the activation of MAPK by Rap and B-Raf, these assays were performed in vitro. Thus, we do not know whether MEK and ERK kinases are physiological downstream targets of Rap and B-Raf in human beta cells. However, glucose and GLP-1 both activate ERK in vivo in isolated human islets, as revealed by phosphorylation at tyrosine 204 (Fig. 2a), which is a good 

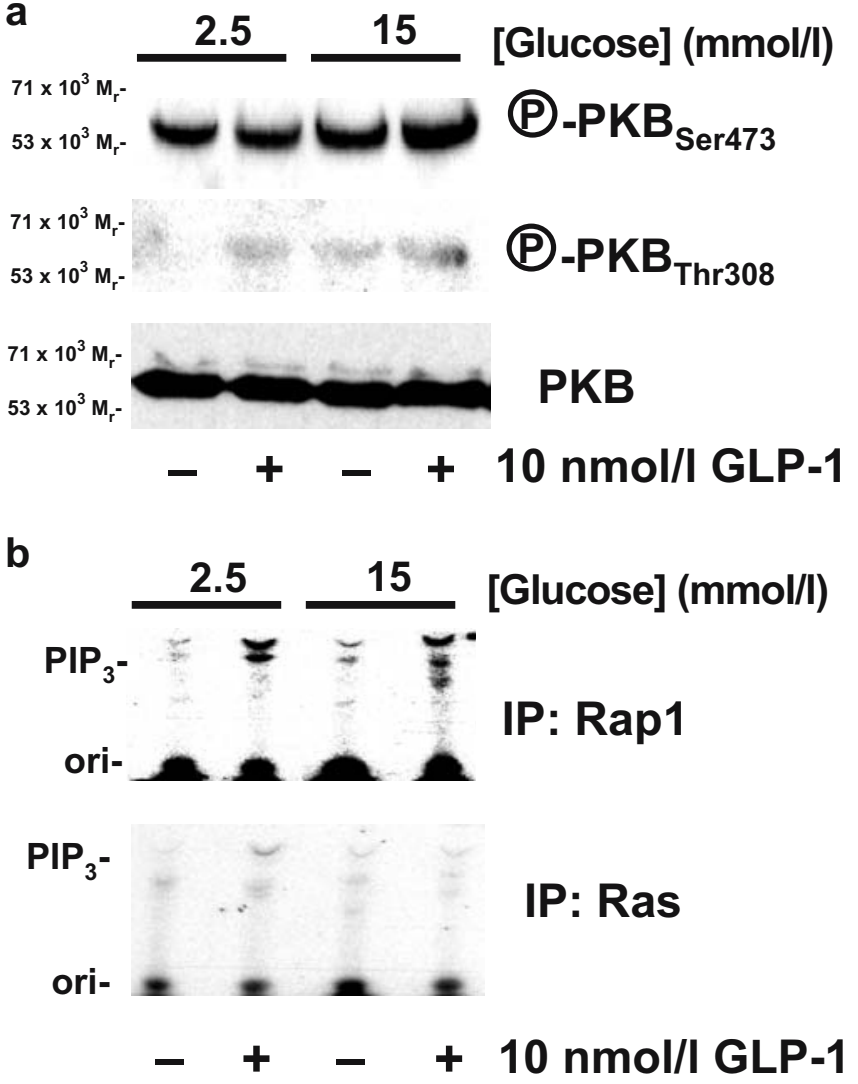

Fig. 4 Glucose and GLP-1 activate PKB and PI3K in human islets. Human islets were equilibrated in 2.5 or $15 \mathrm{mmol} / 1$ glucose for $4 \mathrm{~h}$ and then stimulated with $10 \mathrm{nmol} / 1 \mathrm{GLP}-1$ for $10 \mathrm{~min}$. Detection of phosphorylated PKB (a). Cells were lysed and $10 \mu \mathrm{g}$ of proteins were separated by SDS-PAGE and subjected to immunoblotting with antibodies for phosphorylated PKB. Equal loading was controlled for by immunoblotting for unphosphorylated PKB. PI3K activation is associated with Rap rather than Ras (b). Rap and Ras were immunoprecipitated from islets stimulated with glucose and GLP-1. PI3K assays were performed as described in Materials and methods. The blots shown are representative of three different islet preparations. ori, origin; IP immunoprecipitation; $P I P_{3}$ phosphoinositide 3-phosphate

indicator of ERK activation in beta cells [9]. We also performed MAPK assays with constitutively active Rap and B-Raf (expression vectors RAPG12V and B-RafBxB, respectively) in the beta cell line INS-1E, in which Rap and B-Raf are activated by glucose and GLP-1 in the same manner as in isolated human islets. The results indicated that ERK is activated by active Rap and B-Raf. These effects are partially mediated by guanine nucleotide exchange factors (GEF) that are activated by cAMP, such as cAMPGEFII and C3G-GEF ([2] and D. Ross and D. Hörsch, unpublished results). Thus, Rap and B-Raf are activated by glucose and GLP-1 in isolated human islets and are likely mediators of glucose- and GLP-1-induced MAPK signalling. In contrast, Ras is only slightly activated by glucose and GLP-1, and Raf-1 was not found to be stimulated in isolated human islets. These results imply that Ras and Raf-
1 are not primary mediators of MAPK signalling by glucose and GLP-1 in human beta cells. However, given the demonstrated cooperative hetero-dimerisation of Raf-1 and BRaf [13], Ras and Raf-1 may be important cofactors for the activation of Rap and B-Raf.

The activation of PKB by glucose and GLP-1 in isolated human islets was similar to that observed in INS-1 cells [911]. In our study, the antibody probes detected a lower amount of phosphorylation at threonine 308 than at serine 473 in lysates of human islets. This effect may have been caused by the low amounts of protein used for the immunoblots (usually less than $5 \mu \mathrm{g}$ ), since both antibodies were similarly sensitive when we probed cellular lysates of INS-1 cells using 20-30 $\mu \mathrm{g}$ of protein lysate (results not shown). Alternatively, serine 473 phosphorylation may precede threonine 308 phosphorylation in beta cells. In light of these data, the role of the rictor-mTOR complex should be urgently examined in mammalian beta cells [14].

Using immunoprecipitation, we demonstrated that Rap stimulates PI3K in isolated human islets. Rap-mediated PI3K in islets is similar to Ras-mediated PI3K stimulation in other cellular systems [10] in that it is essentially dependent upon the phosphorylation of $\mathrm{p} 85 \alpha$ by tyrosine kinases (D. Ross and D. Hörsch, unpublished results).

In summary, we have shown that signalling by Rap and $\mathrm{B}-\mathrm{Raf}$ is activated by glucose and GLP-1 in isolated human islets. Rap and B-Raf also mediate glucose- and GLP-1induced ERK signalling in kinase assays in vitro. Furthermore, Rap is an effector for PI3K. Thus, the Rap-B-Raf signalling pathway is a promising therapeutic target in human islet cells that may lead to the development of new treatment modalities for loss of beta cell function and mass in diabetes mellitus.

Acknowledgements This study was supported by grants from the Kempkes Foundation (Marburg, Germany), the Danone Foundation, the German Diabetes Association, The Human Islet Distribution Programme of the Juvenile Diabetes Research Foundation International, and the Federal Ministry of Education and Research (Bundesministerium für Bildung und Forschung; grant project no. 01GN0114).

Duality of interest

The authors are not aware of any conflict of interest.

\section{References}

1. Visboll T, Holst JJ (2004) Incretins, insulin secretion and type 2 diabetes mellitus. Diabetologia 47:357-366

2. Bos JL, de Rooij J, Reedquist KA (2001) Rap1 signaling: adhering to new models. Nat Rev Mol Cell Biol 2:369-377

3. Arnette D, Gibson TB, Lawrence MC et al (2003) Regulation of ERK1 and ERK2 by glucose and peptide hormones in pancreatic beta cells. J Biol Chem 278:32517-32525

4. Briaud I, Lingohr MK, Dickson LM, Wrede CE, Rhodes CJ (2003) Differential activation mechanisms of Erk-1/2 and p70 (S6K) by glucose in pancreatic beta-cells. Diabetes 52:974-983

5. Gomez E, Pritchard C, Herbert TP (2002) cAMP-dependent protein kinase and $\mathrm{Ca}^{2+}$ influx through L-type voltage-gated calcium channels mediate Raf-independent activation of extracellular regulated kinase in response to glucagon-like peptide-1 in pancreatic beta-cells. J Biol Chem 277:48146-48151 
6. Brandhorst H, Brandhorst D, Brendel MD, Hering BJ, Bretzel RG (1998) Improved survival of intraportal pancreatic islet cell allografts in patients with type-1 diabetes mellitus by refined peritransplant management. Cell Transplant 7:489-495

7. Ricordi C, Lacy PE, Finke EH, Olack BJ, Scharp DW (1988) Automated method for isolation of human pancreatic islets. Diabetes 37:413-420

8. Herrmann C, Horn G, Spaargaren M, Wittinghofer A (1996) Differential interaction of the Ras family GTP-binding proteins H-Ras, Rap1 A, and R-Ras with the putative effector molecules Raf kinase and Ral-guanine nucleotide exchange factor. J Biol Chem 271:6794-6800

9. Trümper A, Trümper K, Trusheim H, Arnold R, Göke B, Hörsch D (2001) Glucose-dependent insulinotropic polypeptide is a growth factor for beta (INS-1) cells by pleiotropic signaling. Mol Endocrinol 15:1559-1570
10. Trümper $\mathrm{K}$, Trümper A, Trusheim $\mathrm{H}$, Arnold R, Göke B, Hörsch D (2000) Integrative mitogenic role of protein kinase B/ Akt in beta-cells. Ann N Y Acad Sci 921:242-250

11. Wang Q, Li L, Xu E, Wong V, Rhodes C, Brubaker PL (2004) Glucagon-like peptide-1 regulates proliferation and apoptosis via activation of protein kinase B in pancreatic INS-1 beta cells. Diabetologia 47:478-487

12. Chan TO, Rodeck U, Chan AM et al (2002) Small GTPases and tyrosine kinases coregulate a molecular switch in the phosphoinositide 3-kinase regulatory subunit. Cancer Cell 1:181191

13. Weber CK, Slupsky JR, Kalmes HA, Rapp UR (2001) Active Ras induces heterodimerisation of $\mathrm{cRaf}$ and BRaf. Cancer Res 61:3595-3598

14. Sarbasso DD, Guertin DA, Ali SA, Dabatini DM (2005) Phosphorylation and regulation of Akt/PKB by the rictor-mTOR complex. Science 307:1098-1101 